\title{
NOS2 deficiency has no influence on the radiosensitivity of the hematopoietic system
}

\author{
Chengcheng $\mathrm{Li}^{1,2+}, \mathrm{Yi} \mathrm{LuO}^{2,3 \dagger}$, Lijian Shao ${ }^{2,4}$, Aimin Meng ${ }^{1 *}$ and Daohong Zhou ${ }^{2,4^{*}}$
}

\begin{abstract}
Objective: Previous studies have shown that inhibition of inducible NO synthase (NOS2 or iNOS) with an inhibitor can selectively protect several normal tissues against radiation during radiotherapy. However, the role of NOS2 in ionizing radiation (IR)-induced bone marrow (BM) suppression is unknown and thus was investigated in the present study using $\mathrm{NOS}^{-}{ }^{-}$and wild-type mice 14 days after they were exposed to a sublethal dose of total body irradiation (TBI).

Methods: The effects of different doses of IR (1,2 and $4 \mathrm{~Gy})$ on the apoptosis and colony-forming ability of bone marrow cells from wild-type (WT) and $\mathrm{NOS}^{-}{ }^{-}$mice were investigated in vitro. In addition, we exposed NOS2 ${ }^{-} /^{-}$ mice and WT mice to 6-Gy TBI or sham irradiation. They were euthanized 14 days after TBI for analysis of peripheral blood cell counts and bone marrow cellularity. Colony-forming unit-granulocyte and macrophage, burst-forming unit-erythroid and CFU-granulocyte, erythroid, macrophage in bone marrow cells from the mice were determined to evaluate the function of hematopoietic progenitor cells (HPCs), and the ability of hematopoietic stem cells (HSCs) to self-renew was analysed by the cobblestone area forming cell assay. The cell cycling of HPCs and HSCs were measured by flow cytometry.

Results: Exposure to 2 and 4 Gy IR induced bone marrow cell apoptosis and inhibited the proliferation of HPCs in vitro. However, there was no difference between the cells from WT mice and NOS2 ${ }^{-} /^{-}$mice in response to IR exposure in vitro. Exposure of WT mice and NOS2 ${ }^{-}{ }^{-}$mice to 6 Gy TBI decreased the white blood cell, red blood cell, and platelet counts in the peripheral blood and bone marrow mononuclear cells, and reduced the colony-forming ability of HPCS $(P<0.05)$, damaged the clonogenic function of HSCs. However, these changes were not significantly different in WT and NOS2 ${ }^{-} /{ }^{-}$mice.
\end{abstract}

Conclusion: These data suggest that IR induces BM suppression in a NOS2-independent manner.

Keywords: Hematopoietic stem cell, lonizing radiation, $\mathrm{NOS}^{-{ }^{-}}$

\section{Background}

In the 1940s, Jacobson and his colleagues showed that transplantation of splenocytes and bone marrow cells can rescue the life of mice suffering lethal radiation exposure.

\footnotetext{
*Correspondence: ai_min_meng@126.com; dzhou@uams.edu

${ }^{\dagger}$ Chengcheng Li and Yi Luo contributed equally to this work

${ }^{1}$ Institute of Laboratory Animal Science, Chinese Academy of Medical Sciences (CAMS) and Comparative Medicine Center, Peking Union Medical College (PUMC), Key Laboratory of Human Disease Comparative Medicine, Ministry of Health, Beijing Key Laboratory for Animal Models of Emerging and Remerging Infectious Diseases, Beijing 100021, China ${ }^{4}$ Winthrop P. Rockefeller Cancer Institute, University of Arkansas for Medical Sciences, 4301 W Markham, \#607, Little Rock, AR 72205, USA Full list of author information is available at the end of the article
}

Lorenz et al. published the similar findings after injecting bone marrow cells into the veins of irradiated mice, which were subsequently protected from radiation damage [1]. In the 1960s, Till and McCulloch discovered hematopoietic stem cells, a discovery that revealed why bone marrow transplantation rescues lethal radiationinduced hematopoietic injury. After transplantation, hematopoietic stem cells can regenerate and differentiate into multipotent progenitor cells in lethally irradiated mice and thus promote the recovery of the hematopoietic system in irradiated mice [2-4].

The hematopoietic system differentiates in a hierarchal manner [5]. Hematopoietic stem cells lie at the top 
layer of differentiation and are able to self-renew, proliferate and differentiate into different kinds of blood cells. In the normal environment, hematopoietic stem cells are quiescent and maintain a life-long hematopoietic capacity so that the hematopoietic system cannot be exhausted under stress conditions [6]. Multipotent progenitors (MPPs) and hematopoietic progenitor cells (HPCs) are rapidly proliferating cells but have no self-renewal capacity. Following blood loss, hemolysis or infection, MPPs and HPCs can rapidly proliferate and differentiate into mature blood cells. Meanwhile, the function of the hematopoietic system can be recovered.

Nitric oxide (NO) is a toxic, inorganic gas molecule [7]. $\mathrm{NO}$ is generated by L-arginine and is catalyzed through three types of nitric oxide synthase (NOS) [8]. According to its origin and function, NOS can be divided into the following three types: neuronal nitric oxide synthase (nNOS or NOS1), inducible nitric oxide synthase (iNOS or NOS2) and endothelial nitric oxide synthase (eNOS or NOS3) [9]. Of these, nNOS and eNOS can together be referred to as constitutive nitric oxide synthase (cNOS), as both are constitutively expressed in vivo and are responsible for the synthesis of basic NO. Further, cNOS regulates various physiological functions, including the information transfer between nerves and cells, as well as vasodilation [10]. However, NOS2 is usually not expressed in resting cells. When cells are stimulated by cytokines, physicochemical factors, or immune microorganisms, the expression of NOS2 can be induced. Meanwhile, a non-physiologic concentration of NO can be synthetized through catalysis, and a series of pathological effects are produced [10].

NOS2 is expressed in multiple cells of the bone marrow, including myeloid cells [11], macrophages [12], megakaryocytes, eosinophilic granulocyte and mononuclear cells [13]. In the bone marrow, eNOS can regulate basic $\mathrm{NO}$, and NOS2 also has a significant influence on the generation and function of basic NO [13]. Under physiological conditions, NO can regulate the proliferation, differentiation and mobilization of hematopoietic stem cells [14]. NO can impact the development and differentiation of hematopoietic cells by regulating the formation of red and granular cell colonies [15]. Vilpo et al. found that $\mathrm{NO}$ can reduce the colony-forming ability (CFU-GM) of hematopoietic stem cells and inhibit the growth of $\mathrm{T}$ cells and leukemia cells, findings which indicated that nitric oxide donors have potential applications as antineoplastic drugs [16]. High expression of interferon- $\gamma($ IFN- $\gamma$ ) or tumor necrosis factor- $\beta$ (TNF- $\beta$ ) in patients with aplastic anemia can accelerate NOS2 expression in CD34+ cells and may increase the apoptosis rate of CD34+ cells. NO can also affect the expression of certain genes. NO can induce P53 gene mutation, reduce DNA repair capacity and prompt the premature apoptosis of hematopoietic stem cells $[17,18]$.

Previous studies have shown that ionizing radiation can induce the expression of NOS, including eNOS and NOS2 [19]. In tumor cells, ionizing radiation (IR) can increase the activity of eNOS, promote the generation of intrinsic nitric oxide, and increase murine squamous cell carcinoma (SCCVII) tumor tissue perfusion. Furthermore, IR can cause oxygen enrichment in the tumor tissue and enhance tumor sensitivity to radiotherapy. Deinococcus radiodurans (Drad) are resistant to radiation; the killing effect of UV radiation is enhanced in Drad lacking the nos gene because of defects in NO synthesis [20]. The above results show that NO can enhance radiation sensitivity to some extent. Furthermore, inhibitors of NOS have a protective effect against exposure [21, 22]. Ultraviolet-B (UVB) can induce NOS2 expression, and a specific inhibitor of NOS2, T1023-a, can relieve superficial injury of blood vessels due to radiation [22]. An inhibitor of eNOS and NOS2, T1023, protects against radiation by decreasing circulatory hypoxia, which is a new and promising radiation protection agent. The primary cause is circulatory hypoxia induced by a reduction in the tissue oxygen supply, which is a result of decreased blood flow volume [21]. Our previous study showed using a gene chip that Nos2 was upregulated in HSCs 1 month after 6 Gy total body irradiation (TBI), suggesting that NOS2 may play some effect on IR-induced hematopoietic system injury.

In this study, Nos 2 mRNA expression was measured in sorted LSKs by RT-PCR. Bone marrow cells from $\mathrm{NOS}^{-} /^{-}$mice were obtained to observe the influence of IR on the apoptosis and proliferation of bone marrow cells. Additionally, we investigated whether NOS2 deficiency in mice influences bone marrow and peripheral blood cell count decreases or the reduced number and function of HPC and HSC in response to total-body irradiation (TBI) in order to understand the effect of NOS2 deficiency on radiation injury sensitivity.

\section{Methods \\ Mice}

C57BL/6J and B6.129P2-Nos2tm1Lau/J (NOS2 ${ }^{-1-}$ ) mice were purchased from Jackson Lab (Bar Harbor, MA). Mice were housed at the University of Arkansas for 
Medical Sciences (UAMS) or St. Jude Children's Research Hospital, which are both AAALAC-certified animal facilities. Mice received food and water ad libitum. All mice were used at approximately 8-12 weeks of age. The Institutional Animal Care and Use Committees of UAMS or St. Jude Children's Research Hospital approved all experimental procedures used in this study.

\section{Irradiation of mice}

Mice were exposed to sham irradiation as controls or to a sublethal dose (6 Gy) of TBI in a J.L. Shepherd Model Mark I 137 Cesium $\gamma$-irradiator (J.L. Sheperd, Glendale, CA, USA) at a dose rate of $1.14 \mathrm{~Gy} / \mathrm{min}$ [23]. Dose uniformity was assessed by an independent company (Ashland Specialty Ingredients) with radiographic film and alanine tablets.

\section{Isolation of blood and bone marrow cells}

$\mathrm{NOS} 2^{-1-}$ and WT mice were subjected to a sublethal TBI dose of 6 Gy. On day 14 post-irradiation, peripheral blood was collected (orbital puncture method). Blood parameters were determined by a Hemavet Instrument (Drew Scientific, Inc.). Bone marrow cells (BMCs) were harvested as described previously [24, 25]. The number of viable BMCs was assessed with trypan blue dye exclusion, and counts were determined with a hemocytometer.

\section{Colony-forming unit (CFU) assays for BMCs}

CFU assays were performed with MethoCult GF (M3434, Stem Cell Technologies) according to the manufacturer's instructions as described previously [23, 24].

$\mathrm{NOS} 2^{-I-}$ and WT mice received 6 Gy IR. Bone marrow cells were harvested and placed into 3434 methylcellulose medium 14 days after IR. The CFU for granulocytes and macrophages (CFU-GM) and burst-forming-units for erythroid cells (BFU-E) were measured 7 days later, and the CFUs for granulocytes, erythrocytes, monocytes, and megakaryocytes (GEMM) were measured 12 days later. The colony-forming units were calculated using $10^{5}$ BMCs. Sections with more than 30 cells were scored as a colony under an inverted microscope according to the manufacturer's instructions.

\section{Flow cytometry of HPCs and HSCs after irradiation}

Hematopoietic cell phenotypic analysis was completed by flow cytometry. Briefly, $5 \times 10^{6}$ hematopoietic cells harvested from mouse bone marrow were blocked with Fc-block for $10 \mathrm{~min}$, incubated with biotin-conjugated lineage antibodies (CD4, CD8, CD45R/ B220, Gr-1, Mac-1 and Ter-119) and then stained with streptavidin-FITC (BD Pharmingen), c-KitAPC (BD Pharmingen), and Sca1-PE (eBioscience).
The frequencies of HPCs (Lin-Sca1-c-kit1+cells), LSK cells (Lin-Sca1+c-kit + cells), and HSCs (LinSca1 + c-kit + CD150+CD48-cells) were analyzed with a flow cytometer (LSRII flow cytometer). Data were analyzed using FlowJo software.

\section{Isolation of murine $\mathrm{Lin}^{-} \mathrm{sca}^{-{ }^{+}}{ }^{+} \mathrm{c}-\mathrm{kit}^{+}$cells (LSK cells)}

For the isolation of $\mathrm{Lin}^{-}$cells, BMMNCs were harvested and stained as described above, and the labeled mature lymphoid and myeloid cells were depleted twice by incubation with goat anti-rat IgG paramagnetic beads (Dynal Inc, Lake Success, NY) at a bead-to-cell ratio of approximately 4:1. Cells binding the paramagnetic beads were removed with a magnetic field. The negatively isolated $\mathrm{Lin}^{-}$cells were washed twice with $2 \% \mathrm{FBS} / \mathrm{HBSS}$ and resuspended in complete medium (RPMI-1640 medium supplemented with $10 \% \mathrm{FBS}, 2 \mathrm{mM}$ L-glutamine, $10 \mu \mathrm{M}$ 4-(2-hydroxyethyl)-1-piperazineethanesulfonic acid (HEPES) buffer, $100 \mathrm{U} / \mathrm{ml}$ penicillin, and $100 \mu \mathrm{g} / \mathrm{ml}$ streptomycin; Life Technologies, Grant Island, NY) at $1 \times 10^{6}$ cells $/ \mathrm{ml}$. Lin ${ }^{-}$cells were pre-incubated with antiCD16/32 antibody (BD Biosciences, San Jose, CA) to block Fcy receptors and stained with anti-Sca1-PE and c-Kit-APC antibodies, and then LSK cells were sorted with an Aria II cell sorter (BD Biosciences, San Jose, CA).

\section{Quantitative RT-PCR (qRT-PCR)}

Total cellular RNA was extracted from macrophages using the RNeasy Mini kit (QIAGEN, Gaithersburg, MD). Total cellular RNA was extracted from approximately 5000 sorted LSK cells using the Zymo research QuickRNA Micro Prep kit (The Epigenetics Company, Irvine, CA, USA) according to the manufacturer's instructions. Reverse transcription was performed immediately using Applied Biosystems' High Capacity cDNA Reverse Transcription kit (Life Technologies, Grand Island, NY, USA) according to the manufacturer's instructions. The following Nos2 primers were used: 5-AGCGCTACAACATCC TGGAGGAAGTGG-3; and 5-GTCCATGATGGTCAC ATTCTGCTTCTG-3. GAPDH was used as a housekeeping internal reference for mRNA. The Quantitative PCR conditions were as follows: $95^{\circ} \mathrm{C}$ for $10 \mathrm{~min}, 40 \times\left(95{ }^{\circ} \mathrm{C}\right.$ for $15 \mathrm{~s}$ and $60{ }^{\circ} \mathrm{C}$ for $1 \mathrm{~min}$ ), $95^{\circ} \mathrm{C}$ for $15 \mathrm{~min}, 60^{\circ} \mathrm{C}$ for $60 \mathrm{~min}$, and $95^{\circ} \mathrm{C}$ for $15 \mathrm{~min}$. All reactions were run in triplicate on an ABI StepOnePlus Real-Time PCR System.

\section{Cell cycle}

Lin- cells were stained with antibodies against various HSC cell-surface markers and were then fixed and permeabilized using Fixation-Permeabilization Solution (BD-Pharmingen, San Diego, CA). Subsequently, 
cells were stained with anti-Ki67-FITC and 7-aminoactinomycin $\mathrm{D}$ (7-AAD) and were then analyzed by flow cytometry.

\section{Cobblestone area-forming cell (CAFC) assay}

The CAFC assay for BMCs was done as described previously $[24,26]$.

\section{Statistical analyses}

Data were analyzed by analysis of variance (ANOVA) using GraphPad Prism Software (San Diego, CA). In the event that the ANOVA justified post hoc comparisons between group means, the Newman-Keuls or Tukey's multiple-comparisons test was used. $P<0.05$ was considered significant. Data are presented as the mean \pm SEM.

\section{Results}

Nos2 mRNA expression is upregulated after 6 Gy total body irradiation

LSKs were sorted 1 month after 6 Gy irradiation. PCR results showed that Nos 2 mRNA was increased in the LSKs of irradiated mice compared with wild-type mice (Fig. 1). The Western blots for verifying NOS2 protein levels were not conducted due to very low numbers of cells available after TBI.

\section{NOS2 deficiency does not influence hematopoietic cell apoptosis induced by IR}

Mouse BMMNCs became apoptotic $12 \mathrm{~h}$ after exposure to ionizing radiation at different doses in vitro. The apoptosis rate among hematopoietic progenitor cells increased with increasing radiation doses (Fig. 2A). However, NOS $2^{-} /^{-}$and wild-type mice showed no significant differences in either HSC or HPC at doses of $0,1,2$ or 4 Gy (Fig. 2A, B). This result indicates that NOS2 deficiency does not influence hematopoietic cell apoptosis induced by IR.

We next determined the influence of NOS2 on the inhibition of hematopoietic progenitor cell proliferation due to ionizing radiation in vitro. Ionizing radiation can

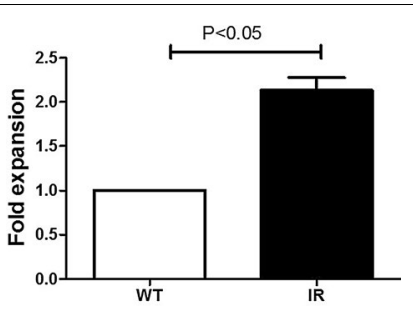

Fig. 1 The mRNA expression of NOS2 were decreased in HSCs. The mRNA of NOS2 was detected by RT-PCR. The results are shown as the mean \pm S.E inhibit the proliferation and differentiation of hematopoietic progenitor cells and reduces the function of hematopoietic progenitor cells. The colony forming unit (CFU) assay is an important indicator used to measure the function of hematopoietic progenitor cells.

The numbers of CFU-GM, BFU-E and CFU-GEMM $(\mathrm{P}<0.05)$ were significantly reduced following 2 and 4 Gy ionizing radiation among mouse bone marrow cells in vitro. The dose of 4 Gy caused more significant damage $(P<0.05$ compared with 0 Gy); however, after receiving different doses of irradiation $(0,1,2$ and 4 Gy), the
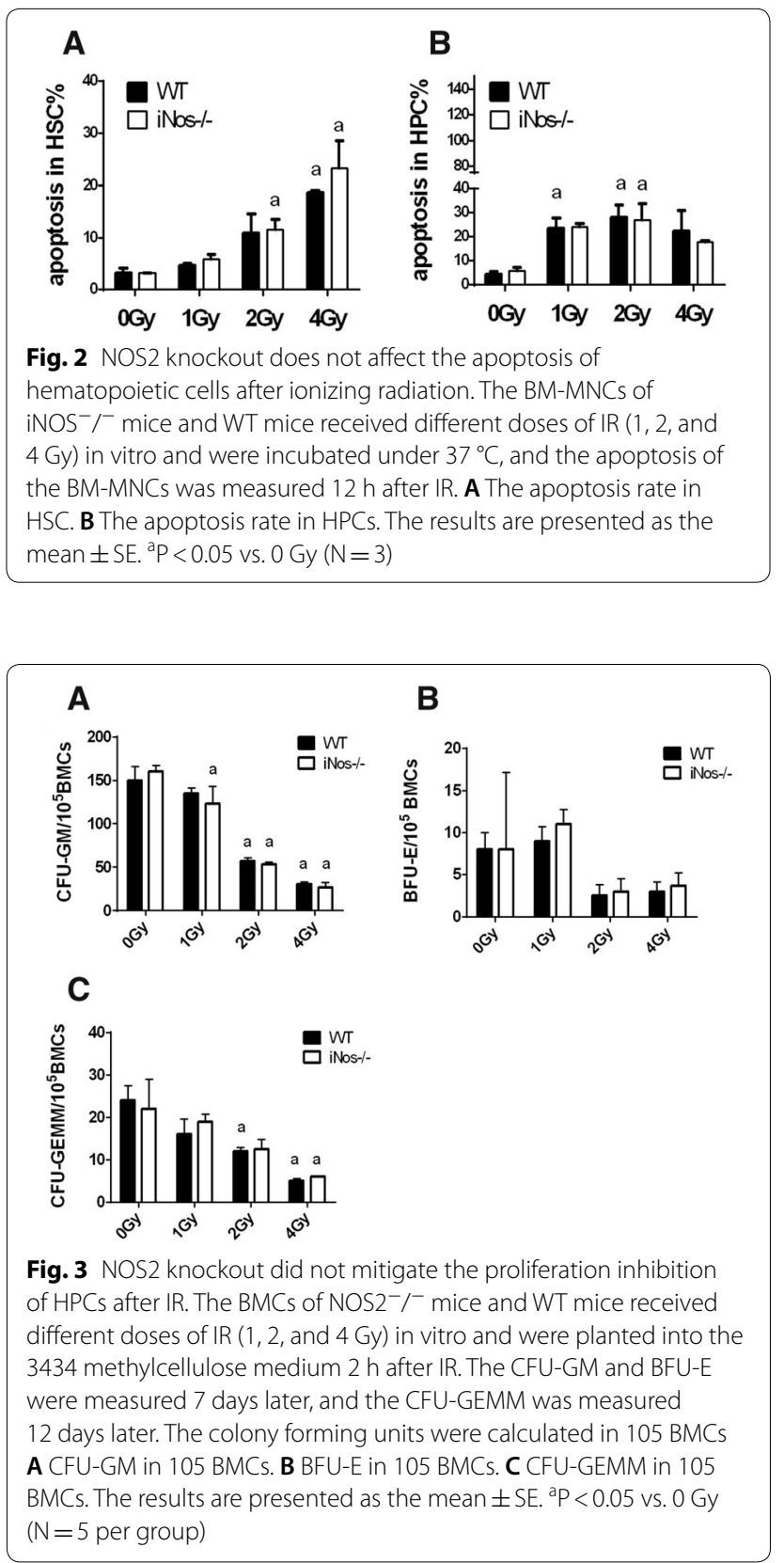
CFU-GM, BFU-E and CFU-GEMM of WT and NOS2 ${ }^{-} /^{-}$ mice in each group were not significantly different $(P>0.05)$. These data indicate that NOS2 deficiency does not affect the apoptosis of hematopoietic cells after ionizing radiation (Fig. 3).

\section{NOS2 deficiency does not influence radiation-induced decreases in peripheral blood cells}

Our previous studies have shown that exposure of C57BL/6J mice to 6 Gy TBI decreases the number of WBCs within 1-2 days after IR. The number of WBCs gradually recoveries from day 14 and almost backs to normal levels by day 30 after TBI. Therefore, in the present study we selected day 14 after IR to examine the role of NOS2 in regulation of the hematopoietic system in response to TBI. The number of mouse peripheral blood was detected 14 days after irradiation. The results are shown in Fig. 4 . In both $\mathrm{NOS}^{-} /^{-}$and WT mice, the number of white blood cells (WBC), red blood cells (RBC), hemoglobin (HGB), and platelets (PLT) in the peripheral blood of mice that received 6 Gy irradiation decreased compared to non-irradiated (0 Gy) mice $(P<0.05)$. When comparing NOS2 ${ }^{-} /^{-}$and WT mice, the number of peripheral blood was not significantly different after receiving 0 or 6 Gy irradiation $(\mathrm{P}>0.05)$, which indicates that NOS2 deficiency does not influence radiation-induced decreases in peripheral blood cells.

\section{NOS2 deficiency does not impact the phenotype of bone marrow cells after irradiation}

To explore the effect of NOS 2 on the radiation sensitivity of the hematopoietic system in mice, the same methods can be adopted as described in "NOS2 deficiency does

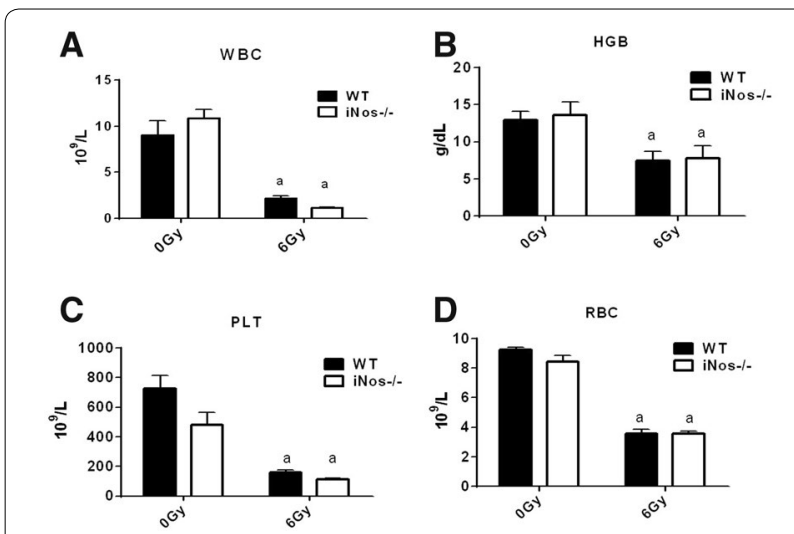

Fig. 4 The impact of NOS2 knockout on the number of PBs after 6 Gy IR. NOS2 ${ }^{-} /^{-}$mice and WT mice received $6 \mathrm{~Gy} I \mathrm{R}$, and the number of PBs were quantified 14 days after IR. A The number of WBCs. B The number of HGBs. C The number of PLTs. D The number of RBCs. The results are presented as the mean $\pm \mathrm{SE}$. ${ }^{\mathrm{P}} \mathrm{P}<0.05 \mathrm{Vs} .0 \mathrm{~Gy}(\mathrm{~N}=5)$

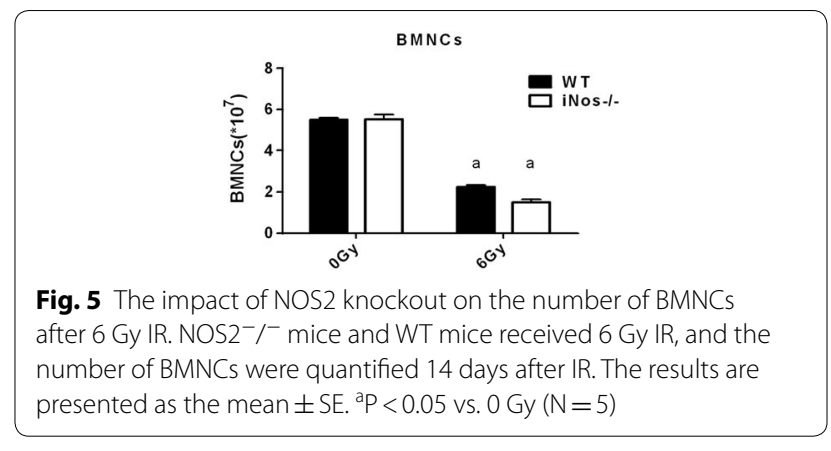

not influence hematopoietic cell apoptosis induced by IR" section. When comparing NOS2 ${ }^{-} /^{-}$and WT mice, the number of bone marrow cells was not significantly different after exposure to 0 or 6 Gy irradiation $(P>0.05$, Fig. 5). In addition, the phenotype of bone marrow cells in mice was detected by flow cytometry 14 days after irradiation. The gating strategy for flow cytometry analysis are shown in Fig. 6A. The proportion and number of hematopoietic progenitor cells (HPC, Lineage-scal-1-ckit+), LSK cells (Lineage-scal-1+ ckit) and hematopoietic stem cells (HSC, Lineage-scal- $1+$ ckit + CD $48+\mathrm{CD} 150+$ ) were examined. The results are shown in Fig. 6B.

Compared to non-irradiated WT mice, the proportion of HPC, LSK and HSC in WT mice after 6 Gy irradiation declined by $39.56,44.85$ and $85.71 \%$, respectively, while the number of HPC, LSK and HSC decreased by 73.64, 76.00 and $93.06 \%$, respectively $(P<0.05)$. Compared to non-irradiated $\mathrm{NOS}^{-} /^{-}$mice, the proportion of HPC, LSK and HSC in $\mathrm{NOS}^{-} l^{-}$mice after 6 Gy irradiation was reduced by $19.85,61.38$ and $52.94 \%(P<0.05)$, respectively, and the number of HPC, LSK and HSC decreased by $79.62,86.00$ and $89.04 \%(P<0.05)$, respectively. These results show that $6 \mathrm{~Gy} \mathrm{TBI}$ in WT or NOS2 ${ }^{-} /^{-}$mice significantly changes the phenotype of bone marrow cells after 14 days. The proportion and number of HPC, LSK and HSCs were reduced, which indicates that hematopoietic system injury is caused by TBI. However, compared to WT mice, the percentage and number of HPC, LSK and HSC in NOS2 $2^{-} /^{-}$mice was not significantly different $(P>0.05)$ after 0 or 6 Gy irradiation exposure. These results indicate that NOS2 deficiency does not affect radiation-mediated decreases in bone marrow cells.

\section{NOS2 deficiency does not influence IR-induced hematopoietic cell function damage}

To determine the effect of NOS2 deficiency on the function of the hematopoietic system, the same methods can be adopted as mentioned in "NOS2 deficiency does not influence hematopoietic cell apoptosis induced by IR" section. Colony forming units (CFUs) and cobblestone 

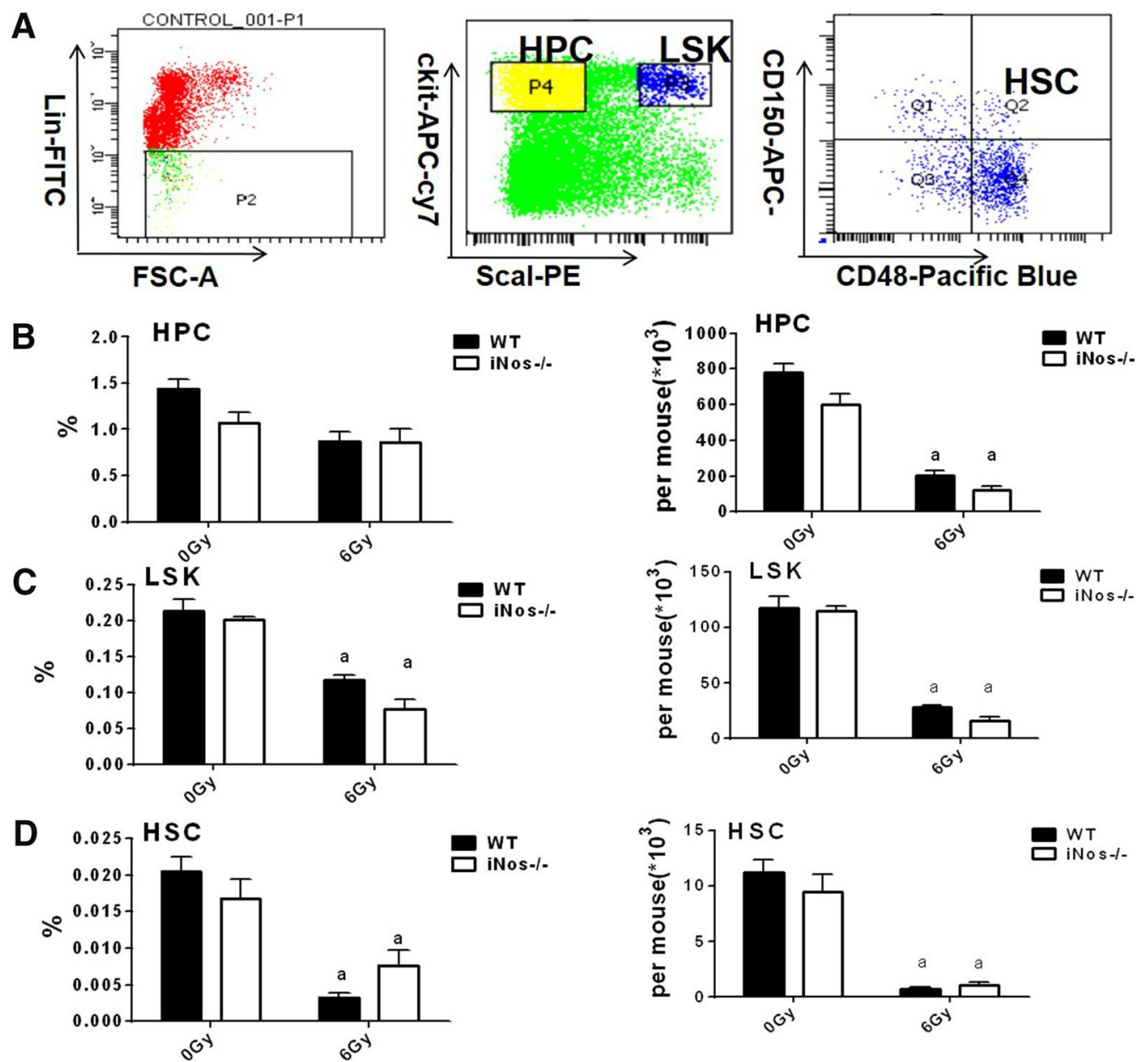

Fig. 6 The impact of NOS2 knockout on the phenotype of BMNCs after 6 Gy IR. NOS2 ${ }^{-}{ }^{-}$mice and WT mice received 6 Gy IR, the bone marrow cells were harvest 14 days after IR and analyzed for phenotype by flow cytometry. A A representative gating strategy of HPCs (lineage-scal-ckit + cells), LSKs (lineage-scal + ckit + cells) and HSCs (lineage-scal + ckit + CD150 + CD48 + cells) was analyzed by flow cytometry; B The frequencies and number of HPCs; $\mathbf{C}$ The frequencies and number of LSKs; $\mathbf{D}$ The frequencies and number of HSCs; the data are presented as the mean $\pm S E M, n=4$

area forming colonies (CAFCs) were measured 14 days after irradiation. The results of the CFU assay can mutually corroborate the results of the CAFC at 2 weeks; the results of CAFC at 5 weeks indicate the self-renewal capability of hematopoietic stem cells, which are related to the results of the transplantation experiment.

The results of the CFU assay show that among WT mice treated with 6 Gy TBI, the CFU-GM number among bone marrow cells was reduced by $78.72 \%(261.6 \pm 10.38$ vs. $55.66 \pm 4.443, P<0.05)$ compared to the non-irradiated group (Fig. 7A). The BFU-E number was reduced by $77.69 \%(17.93 \pm 1.904$ vs. $4.000 \pm 0.577, P<0.05)$ compared to the non-irradiated group (Fig. 7B). The CFUGEMM number was reduced by $85.71 \%(9.666 \pm 1.224$ vs. $1.388 \pm 0.369, \mathrm{P}<0.05)$ compared to the non-irradiated group (Fig. 7C). Compared to non-irradiated NOS2 ${ }^{-} /^{-}$ mice, the CFU-GM, BFU-E and CFU-GEMM among $\mathrm{NOS} 2^{-} /^{-}$mice that received 6 Gy radiation were reduced by $84.76 \%(240.6 \pm 14.43$ vs. $36.66 \pm 2.651, P<0.05)$, $84.44 \%(17.49 \pm 0.926$ vs. $2.722 \pm 0.611, P<0.05)$ and $91.97 \%(9.000 \pm 1.452$ vs. $0.7222 \pm 0.264, P<0.05)$. Our results show that ionizing radiation at a dose of 6 Gy can significantly reduce the proliferation of hematopoietic progenitor cells. However, when WT mice are compared to NOS2 $2^{-} /^{-}$mice, hematopoietic cell apoptosis and function are not significantly different at 0 or 6 Gy irradiation, which indicates that NOS2 deficiency has no influence IR-induced hemopoietic progenitor cell damage.

The cobblestone area forming cell (CAFC) assay can evaluate the function of hematopoietic stem cells and 

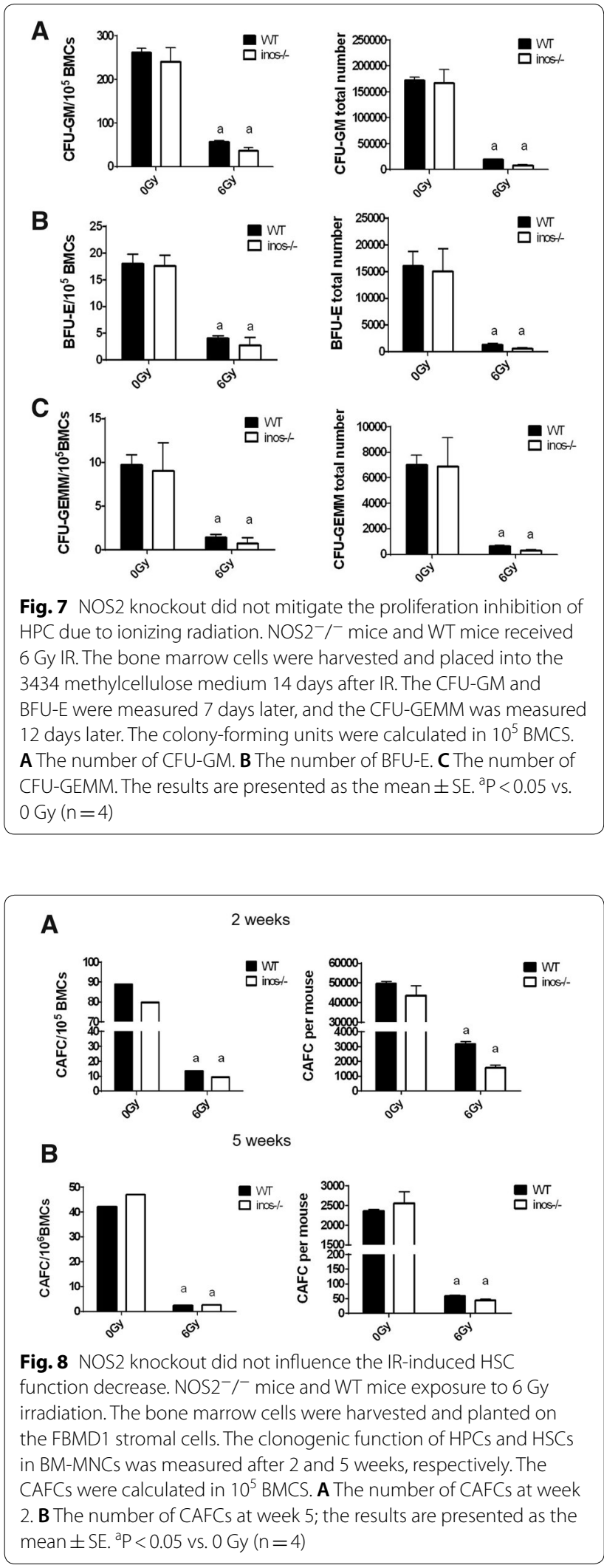

hematopoietic progenitor cells in vitro. Bone marrow cells from WT mice and $\mathrm{NOS}^{-} /^{-}$mice were exposed to 0 or 6 Gy TBI and were then plated on FBMD1 stromal cells. CAFCs were detected after 2 and 5 weeks to measure the function of hematopoietic progenitor cells and hematopoietic stem cells. The results of CAFC for 2 and 5 weeks show that 6 Gy TBI reduced the function of hematopoietic stem cells and hematopoietic progenitor cells in WT and NOS2 $2^{-} /^{-}$mice. However, there was no difference between the WT and $\mathrm{NOS}^{-} l^{-}$mice after receiving 0 or 6 Gy irradiation (Fig. 8A, B), which shows that NOS2 deficiency does not influence hematopoietic stem cell function damage after irradiation.

\section{NOS2 deficiency does not impact IR-induced cell cycle arrest}

To observe the effect of NOS2 deficiency on the homeostasis of hematopoietic cells, WT and $\mathrm{NOS}^{-} /^{-}$mice were exposed to 0 or 6 Gy TBI. The cell cycle status of hematopoietic cells was then detected. For irradiated $\mathrm{WT}$ and $\mathrm{NOS} 2^{-} /^{-}$mice, the percentages of cells in the G0 period were as follows: HSC, 64.1 and $63.6 \%$, respectively; LSK, 46.1 and 56.6\%, respectively; and HPC, 3.8 and $5.9 \%$, respectively (Fig. 9B). These data show that a small number of cells at a higher degree of differentiation will be in G0. Conversely, the proportion of cells in SG2-M phase will be larger. The percentage of HPC, MPP and HSCs in G0 was lower after 6 Gy TBI. Meanwhile, the proportion in S-G2-M was significantly increased, which indicates that TBI induces hematopoietic cells to cycle. However, when comparing WT and NOS2 $2^{-}$ mice, there was no significant difference after either 0 or 6 Gy IR. Thus, NOS2 deficiency does not influence the cell cycle change that is induced by TBI.

\section{Discussion}

Exposure to IR leads to increased oxidative stress, DNA damage, genomic instability and increased inflammation; NOS2 is implicated in regulation of these processes [27-30], but its role in radiation responses has not been investigated. Gene chip results showed that Nos2 is upregulated in HSCs 1 month after 6 Gy TBI (data not shown), and this result has been further verified by RTPCR using sorted LSKs. The present study examined the role of NOS2 in response to IR using a NOS2-deficient mouse model.

Our results showed that 1,2 , and 4 Gy irradiation induces defects in apoptosis and colony forming ability among hematopoietic progenitor cells in $\mathrm{NOS}_{2}^{-} /^{-}$and WT mice in vitro. There were no significant differences between NOS2 $2^{-} /^{-}$and the WT group, suggesting that the NOS2 deficiency does not affect the sensitivity of hematopoietic cells in vitro. Michurina et al. found that 

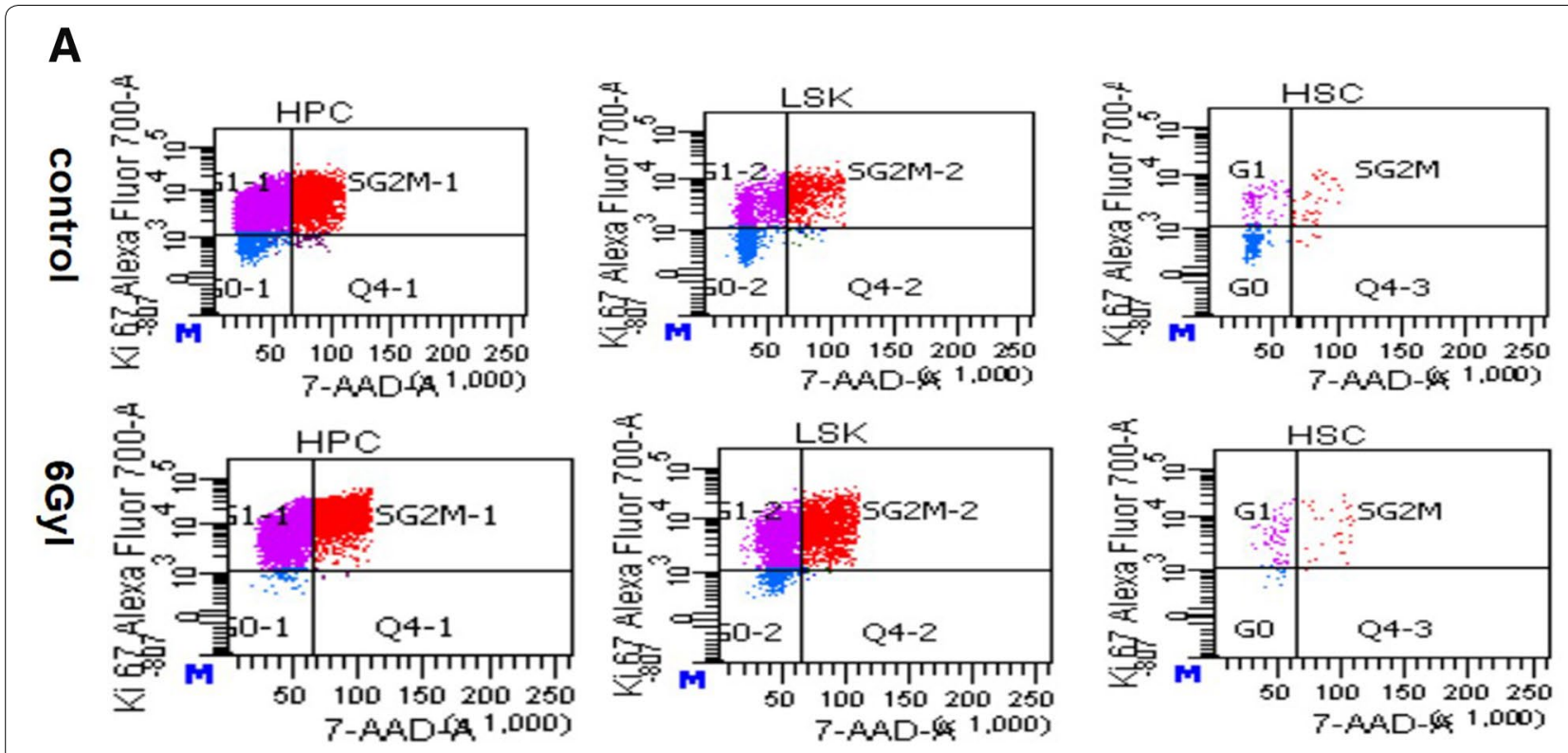

B

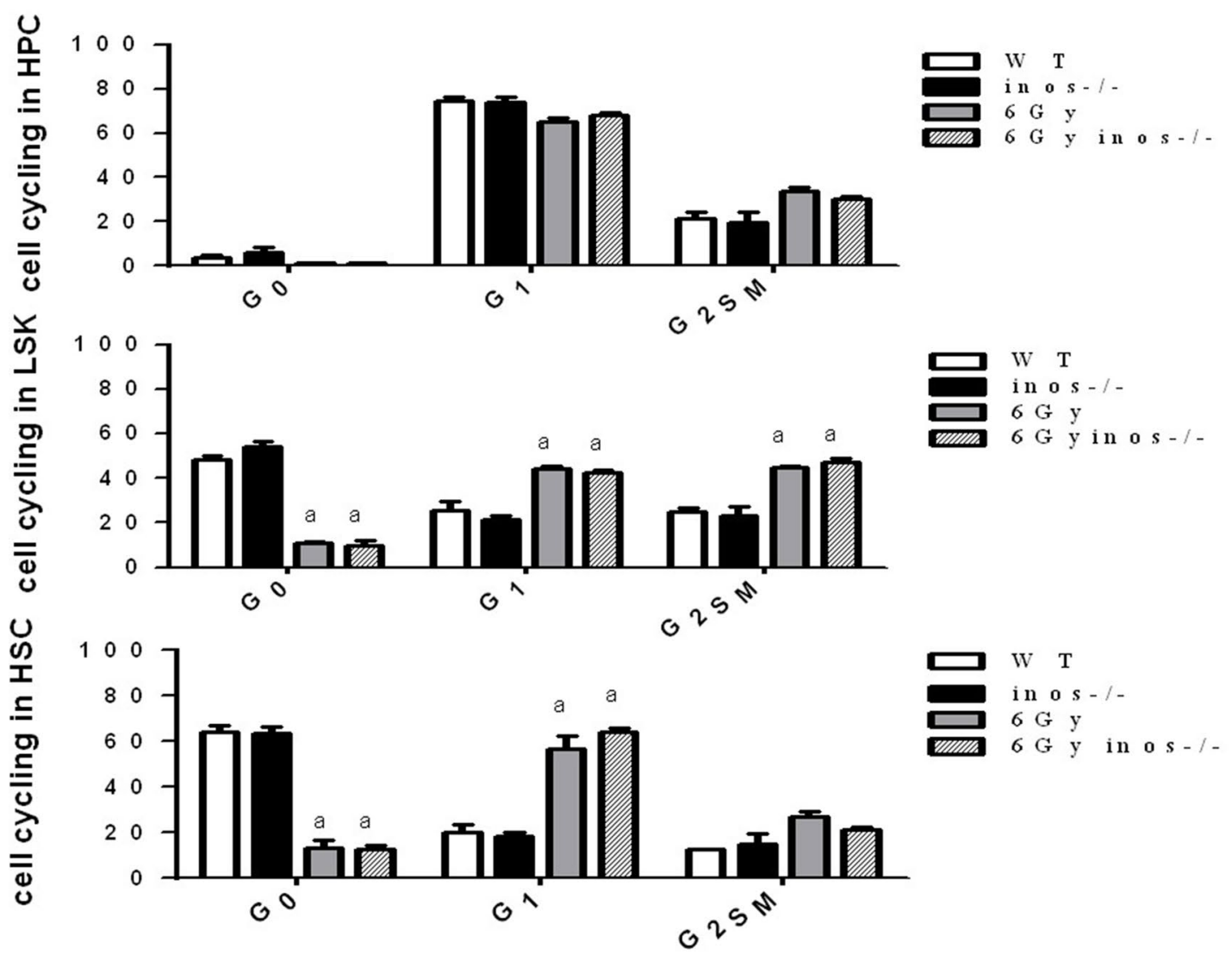

Fig. 9 NOS2 knockout has no influence in the cell cycling arrest of BMNCs after 6 Gy ionizing radiation. NOS2- ${ }^{-}$mice and WT mice received 6 Gy IR. and analyzed for cell cycling by flow cytometry. A A representative gating strategy of cell cycling was analyzed by flow cytometry; $\mathbf{B}$ The analysis of cell cycling in HPCs, LSKs and HSCs. The data are presented as the mean \pm SEM $(n=4)$ 
NOS inhibitors do not affect the apoptosis and survival of hematopoietic stem cells in vitro [13]. Our results showed that the apoptosis and CFU of bone marrow cells in NOS2 $2^{-} /^{-}$mice showed no significant difference compared with that of WT mice after exposure to 1,2 , and 4 Gy TBI. These results demonstrated that physiological NO does not affect the function of hematopoietic cells in vitro. One reason may be because a relatively small amount of $\mathrm{NO}$ production is induced in hematopoietic cells by NOS2 under the physiological state [13]. Early studies showed that low concentrations of NO donor decreased the CFU ability of CD34+ cells in human bone marrow [15], suggesting that exogenous NO can damage the differentiation of hematopoietic cells.

To explore the effect of NOS2 on the hematopoietic system, WT and NOS2 ${ }^{-} /^{-}$mice received sham irradiation (0 Gy) and 6 Gy TBI; the results showed that 6 Gy TBI leads to decreased numbers of peripheral blood cells and bone marrow cells, damages the function of HSPCs, and breaks hematopoietic stem cell homeostasis in WT mice. HSCs under resting state are important for resisting harmful stimuli, maintaining stemness, and preventing HSCs from premature exhaustion [31]. The NOS2 ${ }^{-} /^{-}$mice showed the same results after 6 Gy TBI. Previous studies showed that NO induce cell cycle arrest by regulating the cell cycle protein dependent kinase inhibitor p21WAF1 [32]. To inhibit NOS activity, we can increase the number and self-renewal capacity of hematopoietic stem cells [13]. Our results showed that 6 Gy TBI induces bone marrow cell entry into the cell cycle in $\mathrm{NOS}^{-} /^{-}$mice; HSC went from the G0 phase to G2SM phase, while there was no significant difference between WT mice and NOS2 $2^{-} /^{-}$mice.

The present study showed that induction of HSC damage by IR is independent of NOS2, because NOS2 deficiency in mice had no significant effects on IR-induced bone marrow cell number and colony forming ability. Furthermore, the lack of an effect of NOS2 deficiency cannot be attributed to the cell cycling arrest induced by IR. These data suggest IR induces BM suppression in a NOS2-independent manner, even though NOS2 is upregulated in HSCs after 6 Gy TBI.

Previous studies have shown that LPS, TNF $\alpha$, IL-17 and other modulators can induce the activation of $\mathrm{NFK}_{\mathrm{K}} \mathrm{B}$ and MAPK in bone marrow; therefore, NOS2 expression can be stimulated, sufficient NO can be produced, and the function of bone marrow cells can be reduced. High expression IFN- $\gamma$ of TNF- $\beta$ in the bone marrow cells of aplastic anemia patients can accelerate NOS2 expression of CD34+ cells and result in the apparent increase in CD34+ cell apoptosis rate. However, IR-induced NOS2 activation in bone marrow cells is seldom researched; a previous study showed that the specific inhibitor of
NOS2, T1023-a, can relieve superficial injury of blood vessels due to radiation [33], which has a protective effect against radiation by decreasing circulatory hypoxia. In our study, NOS2 expression was inhibited by NOS 2 deficiency, which did not rescue IR-induced hematopoietic system damage.

Reactive oxygen species (ROS) induced by IR could damage HSCs by interfering with p38MAPK [34]. Even though $\mathrm{T}$ lymphocyte dysfunction has been reported to be attributable to nitrative stress induced by reactive nitrogen species (RNS) [35], there is still no evidence to demonstrate that HPCs and HSCs can be damaged by IRinduced RNS. A previous study showed that LPS induces oxidative DNA damage in the bone marrow of mice in an NOS2-dependent pathway [36], suggesting that the oxidative stress induced by IR may be different from that induced by inflammation.

However, whether the reactive nitrogen species (RNS) induced by NOS2 after ionizing radiation in the hematopoietic system shows pathological injury remains to be investigated [37]. LPS, TNF $\alpha$ and IL-17 induce the expression of NOS2 by activating NFKB and MAPK [38, 39]; NO could damage bone marrow function, suggesting that the oxidative stress induced by IR is different from that induced by inflammation. It is possible that the increased production of reactive active oxygen (ROS) and activation of p38MAPK as reported previously [34, 40]. In addition, activation of NFkB was also reported to be involved in IR-induced bone marrow suppression [41]. Furthermore, HSCs from p53-deficient mice are less sensitive to IR than are those from wild-type mice and treatment with a p53 inhibitor protected mice from IR induced lethal damage by suppression of p53-dependent apoptosis [42]. Finally, activation of the G-CSF/Stat3/ BATF pathway can also contribute to IR-induced HSC injury via promoting HSC differentiation [5].

In addition, IR damages the hematopoietic system function through multiple pathways as follows: IR can induce DNA damage, resulting in cell apoptosis; IR can inhibit hematopoietic stem cell differentiation; and IR can activate oxidative stress in stem or progenitor cells and promote senescence $[5,43]$. As a consequence, the damaging effect of these pathways may compensate for the cytotoxic effects caused by NOS2 deficiency.

Studies in knockout mouse models of p53, targets of p53, CCAAT/Enhancer-Binding Protein Delta (C/ebpd) and other genes led to the identification of some of the molecular mechanisms that drive cellular, tissue, and organismal responses to radiation [23, 44, 45]. However, additional mechanisms that contribute to the radiation response remain undefined, and investigations in this direction will aid in the development of novel, targetspecific interventions to protect normal tissues from 
radiation injury [46]. Christopher et al. reported that NOS2a acts downstream of the transcription factor C/ ebpb to control expansion of HSPCs following infection [47]. Our study suggested that manipulating NOS2 activity does not influence the capacity of mammalian HSPCs. In the future, a model with hematopoietic system-specific NOS2 deficiency may help to exclude the compensation effect of other NOS enzymes. The present study showed that IR induced BM suppression in a NOS2-independent manner at 14 days after TBI, the delayed changes need further investigation.

\section{Conclusion}

The present study showed that IR induces BM suppression in a NOS2-independent manner, even though NOS2 is upregulated in HSCs after 6 Gy TBI.

\begin{abstract}
Abbreviations
BM: bone marrow; BMMNCs: bone marrow mononuclear cells; BFU-E: burst-forming unit erythroid; CFU-GM: colony forming unit-granulocyte, macrophage; CFU-GEMM: colony forming unit-granulocyte, erythroid, macrophage, megakaryocyte; CAFC: cobblestone area-forming cell assay; cNOS: constitutive nitric oxide synthase; DNA: deoxyribonucleic acid; DPI: diphenylene iodonium; DMSO: dimethyl sulfoxide; eNOS: endothelial nitric oxide synthase; FBS: fetal bovine serum; FITC: fluorescein isothiocyanate; FBMD-1: flask bone marrow dexter-1; HGB: hemoglobin; HPCs: hematopoietic progenitor cells; HSCs: hematopoietic stem cells; IR: ionizing radiation; NOS2: inducible nitric oxide synthase; IFN-ү: interferon-gamma; Lin-BMCs: lineagenegative bone marrow cells; LYM: lymphocyte; LPS: lipopolysaccharide; MFI: mean fluorescence intensity; MPP: multipotent progenitor; MAPK: mitogen activated protein kinases; NF-kB: nuclear factor-kappa beta; NOS: nitric oxide synthase; nNOS: neuronal nitric oxide synthase; p38MAPK: p38 mitogen activated protein kinases; PI: propidium iodide; PLT: platelet; ROS: reactive oxygen species; RT-PCR: reverse transcriptase-polymerase chain reaction; qRTPCR: quantitative RT-PCR; RBC: red blood cell; TBI: total body irradiation; TNF- $\beta$ : tumor necrosis factor-beta; WT: wild-type; WBC: white blood cell.
\end{abstract}

\section{Authors' contributions}

LCC and LY designed, performed and analyzed most of the experiments and wrote the manuscript. S $\amalg$ performed some experiments. MAM conceived, designed, and analyzed data. ZD conceived, designed, and supervised the study and analyzed and interpreted data. All authors read and approved the final manuscript.

\section{Author details \\ ${ }^{1}$ Institute of Laboratory Animal Science, Chinese Academy of Medical Sciences (CAMS) and Comparative Medicine Center, Peking Union Medical College (PUMC), Key Laboratory of Human Disease Comparative Medicine, Ministry of Health, Beijing Key Laboratory for Animal Models of Emerging and Remerging Infectious Diseases, Beijing 100021, China. ${ }^{2}$ Department of Pharmaceutical Sciences, University of Arkansas for Medical Sciences, Little Rock, AR, USA. ${ }^{3}$ Department of Hematology, Tongji Hospital, Tongji Medical College, Huazhong University of Science and Technology, Wuhan 430030, China. ${ }^{4}$ Winthrop P. Rockefeller Cancer Institute, University of Arkansas for Medical Sciences, 4301 W Markham, \#607, Little Rock, AR 72205, USA.}

\section{Acknowledgements \\ We thank Feng Wei and Chang Jianhui for their analysis of flow cytometry data.}

\section{Competing interests}

The authors declare that they have no competing interests.

\section{Availability of data and materials}

The datasets used and/or analyzed during the current study are available from the corresponding author upon request.

\section{Consent for publication}

Not applicable.

\section{Ethics approval and consent to participate}

The Institutional Animal Care and Use Committees of UAMS or St. Jude Children's Research Hospital approved all animal experimental procedures used in this study.

\section{Funding}

This study was supported by grants from the National Institutes of Health (R01-CA122023 and A1080421). This study was also supported by National Science Foundation for Young Scientists of China (81703170) and CAMS Innovation Fund for Medical Sciences (CIFMS) WY (2017-12M-3-015).

\section{Publisher's Note}

Springer Nature remains neutral with regard to jurisdictional claims in published maps and institutional affiliations.

Received: 21 January 2018 Accepted: 12 April 2018

Published online: 27 April 2018

\section{References}

1. Lorenz E, Uphoff D, Reid TR, Shelton E. Modification of irradiation injury in mice and guinea pigs by bone marrow injection. Radiology. 1952;58(6):863.

2. Becker AJ, Mcculloch EA, Till JE. Cytological demonstration of the clonal nature of spleen colonies derived from transplanted mouse marrow cells. Nature. 1963;197(11):452-4.

3. Mcculloch EA, Je T. The radiation sensitivity of normal mouse bone marrow cells, determined by quantitative marrow transplantation into irradiated mice. Radiat Res. 1960;13(1):115-25.

4. Till JE, Mcculloch EA. A direct measurement of the radiation sensitivity of normal mouse bone marrow cells. Radiat Res. 1961;14(2):213-22.

5. Shao L, Yi L, Zhou D. Hematopoietic stem cell injury induced by ionizing radiation. Antioxid Redox Signal. 2014;20(9):1447.

6. Wilson A, Laurenti E, Trumpp A. Balancing dormant and self-renewing hematopoietic stem cells. Curr Opin Genet Dev. 2009;19(5):461.

7. Ignarro LJ, Buga GM, Wood KS, Byrns RE, Chaudhuri G. Endotheliumderived relaxing factor produced and released from artery and vein is nitric oxide. Proc Natl Acad Sci USA. 1987;84(24):9265.

8. Leifeld L, Fielenbach M, Dumoulin FL, Speidel N, Sauerbruch T, Spengler $U$. Inducible nitric oxide synthase (iNOS) and endothelial nitric oxide synthase (eNOS) expression in fulminant hepatic failure. J Hepatol. 2002;37(5):613-9.

9. Wang W, Lee Y, Lee CH. Effects of nitric oxide on stem cell therapy. Biotechnol Adv. 2015;33(8):1685.

10. Aktan F. iNOS-mediated nitric oxide production and its regulation. Life Sci. 2004;75(6):639-53.

11. Pu S, Qin B, He H, Zhan J, Wu Q, Zhang X, et al. Identification of early myeloid progenitors as immunosuppressive cells. Sci Rep. 2016;6:23115.

12. Aleksinskaya MA, van Faassen EE, Nelissen J, Janssen BJ, De Mey JG, Hanemaaijer R, Rabelink T, van Zonneveld AJ, et al. Identification of free nitric oxide radicals in rat bone marrow: implications for progenitor cell mobilization in hypertension. PLoS ONE. 2013;8(3):e57761.

13. Michurina T, Krasnov P, Balazs A, Nakaya N, Vasilieva T, Kuzin B, et al. Nitric oxide is a regulator of hematopoietic stem cell activity. Mol Ther. 2004;10(2):241-8.

14. Aicher A, Heeschen C, MildnerRihm C, Urbich C, Ihling C, Technaulhling $K$, et al. Erratum: essential role of endothelial nitric oxide synthase for mobilization of stem and progenitor cells. Nat Med. 2003;9(11):1370.

15. Shami PJ, Weinberg JB. Differential effects of nitric oxide on erythroid and myeloid colony growth from CD34+ human bone marrow cells. Blood. 1996;87(3):977. 
16. Vilpo JA, Vilpo LM, Vuorinen P, Moilanen E, Metsäketelä T. Mode of cytostatic action of mesoionic oxatriazole nitric oxide donors in proliferating human hematopoietic cells. Anticancer Drug Des. 1997;12(2):75-89.

17. Weinberg JB. Nitric oxide and life or death of human leukemia cells. In: Bonavida B, editor. Nitric oxide (NO) and cancer. New York: Springer; 2010. p. 147-67.

18. Hickok JR, Divya V, Kate J, Thomas DD. Oxygen dependence of nitric oxide-mediated signaling. Redox Biol. 2013;1 (1):203-9.

19. Ryu YK, Lee MH, Lee J, Lee JW, Jang SJ, Kang JH, et al. Irradiated cancer cells promote tumor growth by activation of Toll-like receptor 1-mediated inducible nitric oxide synthase in macrophages. J Leukoc Biol. 2015;97(4):711.

20. Hansler A, Chen Q, Ma Y, Gross SS. Untargeted metabolite profiling reveals that nitric oxide bioynthesis is an endogenous modulator of carotenoid biosynthesis in Deinococcus radiodurans and is required for extreme ionizing radiation resistance. Arch Biochem Biophys. 2016;589:38.

21. Filimonova MV, Shevchenko LI, Trofimova TP, Makarchuk VM, Shevchuk AS, Lushnikova GA. On the mechanism of radioprotective effect of NOsynthase inhibitors. Radiats Biol Radioecol. 2014;54(5):500-6.

22. Santa-Cruz DM, Pacienza NA, Zilli CG, Tomaro ML, Balestrasse KB, Yannarelli GG. Nitric oxide induces specific isoforms of antioxidant enzymes in soybean leaves subjected to enhanced ultraviolet-B radiation. J Photochem Photobiol B Biol. 2014;141:202.

23. Pawar SA, Shao L, Chang J, Wang W, Pathak R, Zhu X, et al. C/EBPS Deficiency Sensitizes Mice to lonizing Radiation-Induced Hematopoietic and Intestinal Injury. PLoS ONE. 2014;9(4):e94967.

24. Chang J, Wang Y, Shao L, Laberge RM, Demaria M, Campisi J, et al. Clearance of senescent cells by ABT263 rejuvenates aged hematopoietic stem cells in mice. Nat Med. 2016;22(1):78.

25. Li C, Lu L, Zhang J, Huang S, Xing Y, Zhao M, et al. Granulocyte colonystimulating factor exacerbates hematopoietic stem cell injury after irradiation. Cell Biosci. 2015;5(1):65.

26. Meng A, Wang Y, Zant GV, Zhou D. lonizing radiation and busulfan induce premature senescence in murine bone marrow hematopoietic cells. Cancer Res. 2003:63(17):5414-9.

27. Flaherty RL, Owen M, Fagan-Murphy A, Intabli H, Healy D, Patel A, et al. Glucocorticoids induce production of reactive oxygen species/reactive nitrogen species and DNA damage through an iNOS mediated pathway in breast cancer. Breast Cancer Res. 2017;19(1):35.

28. Sun SJ, Wu XP, Song HL, Li GQ. Baicalin ameliorates isoproterenol-induced acute myocardial infarction through iNOS, inflammation, oxidative stress and P38MAPK pathway in rat. Int J Clin Exp Med. 2015;8(12):22063.

29. Kiang JG, Jiao W, Cary LH, Mog SR, Elliott TB, Pellmar TC, et al. Wound trauma increases radiation-induced mortality by activation of iNOS pathway and elevation of cytokine concentrations and bacterial infection. Radiat Res. 2010;173(3):319-32.

30. Kiang JG, Krishnan S, Lu X, Li Y. Inhibition of inducible nitric-oxide synthase protects human T cells from hypoxia-induced apoptosis. Mol Pharmacol. 2008;73(3):738-47.

31. Wang Z, Ema H. Mechanisms of self-renewal in hematopoietic stem cells. Int J Hematol. 2016;103(5):498-509.
32. Yang J, Wu LJ, Tashino S, Onodera S, Ikejima T. Protein tyrosine kinase pathway-derived ROS/NO productions contribute to G2/M cell cycle arrest in evodiamine-treated human cervix carcinoma HeLa cells. Free Radical Res. 2010;44(7):792-802.

33. Filimonova MV, Ulyanenko SE, Shevchenko LI, Kuznetsova MN, Makarchuk VM, Chesnakova EA, et al. Radioprotective properties of NO-synthase inhibitor T1023: II. The ability for selective protection of normal tissues during radiotherapy of tumors. Radiat Biol Radioecol. 2015;55(3):260.

34. Wang Y, Liu L, Zhou D. Inhibition of p38 MAPK attenuates ionizing radiation-induced hematopoietic cell senescence and residual bone marrow injury. Radiat Res. 2011;176(6):743-52.

35. Kasic T, Colombo P, Soldani C, Wang CM, Miranda E, Roncalli M, et al. Modulation of human T-cell functions by reactive nitrogen species. Eur J Immunol. 2011;41(7):1843-9.

36. Vestergaard S, Loft S, Møller P. Role of inducible nitrogen oxide synthase in benzene-induced oxidative DNA damage in the bone marrow of mice. Free Radical Biol Med. 2002;32(5):481.

37. Vlaskilafarge M, Ivanovic Z. Reliability of ROS and RNS detection in hematopoietic stem cells - potential issues with probes and target cell population. J Cell Sci. 2015;128(21):3849.

38. Chan ED, Riches DWH. IFN- $\gamma+$ LPS induction of iNOS is modulated by ERK, JNK/SAPK, and p38mapk in a mouse macrophage cell line. Am J Physiol Cell Physiol. 2001;280(3):C441.

39. Salim T, Sershen CL, May EE. Investigating the role of TNF- $a$ and IFN- $\gamma$ activation on the dynamics of iNOS gene expression in LPS stimulated macrophages. PLoS ONE. 2016;11(6):e0153289.

40. Li D, Wang Y, Wu H, Lu L, Wang $X$, Zhang J, et al. The effects of p38 MAPK inhibition combined with G-CSF administration on the hematoimmune system in mice with irradiation injury. PLoS ONE. 2013;8(4):e62921.

41. Xiao M, Inal CE, Parekh VI, Li XH, Whitnall MH. Role of NF-kappaB in hematopoietic niche function of osteoblasts after radiation injury. Exp Hematol. 2009;37(1):52-64.

42. Komarov PG, Komarova EA, Kondratov RV, Christovtselkov K, Coon JS, Chernov MV, et al. A chemical inhibitor of p53 that protects mice from the side effects of cancer therapy. Science. 1999;285(5434):1733-7.

43. Wang Y, Schulte BA, Zhou D. Hematopoietic stem cell senescence and long-term bone marrow injury. Cell Cycle 2006,5:35-8.

44. Komarova EA, Kondratov RV, Wang K, Christov K, Golovkina TV, Goldblum $J R$, et al. Dual effect of p53 on radiation sensitivity in vivo: $\mathrm{p} 53$ promotes hematopoietic injury, but protects from gastro-intestinal syndrome in mice. Oncogene. 2004;23(19):3265-71.

45. Shao L. Deletion of proapoptotic Puma selectively protects hematopoietic stem and progenitor cells against high-dose radiation. Blood. 2010;115(23):4707.

46. Prasanna PGS, Stone HB, Wong RS, Capala J, Bernhard EJ, Vikram B, et al. Normal tissue protection for improving radiotherapy: where are the gaps? Transl Cancer Res. 2012;1 (1):35.

47. Hall CJ, Flores MV, Oehlers SH, Sanderson LE, Lam EY, Crosier KE, et al. Infection-responsive expansion of the hematopoietic stem and progenitor cell compartment in zebrafish is dependent upon inducible nitric oxide. Cell Stem Cell. 2012;10(2):198-209.

Ready to submit your research? Choose BMC and benefit from

- fast, convenient online submission

- thorough peer review by experienced researchers in your field

- rapid publication on acceptance

- support for research data, including large and complex data types

- gold Open Access which fosters wider collaboration and increased citations

- maximum visibility for your research: over $100 \mathrm{M}$ website views per year

At BMC, research is always in progress.

Learn more biomedcentral.com/submissions 